\title{
In situ microCT for planetary exploration
}

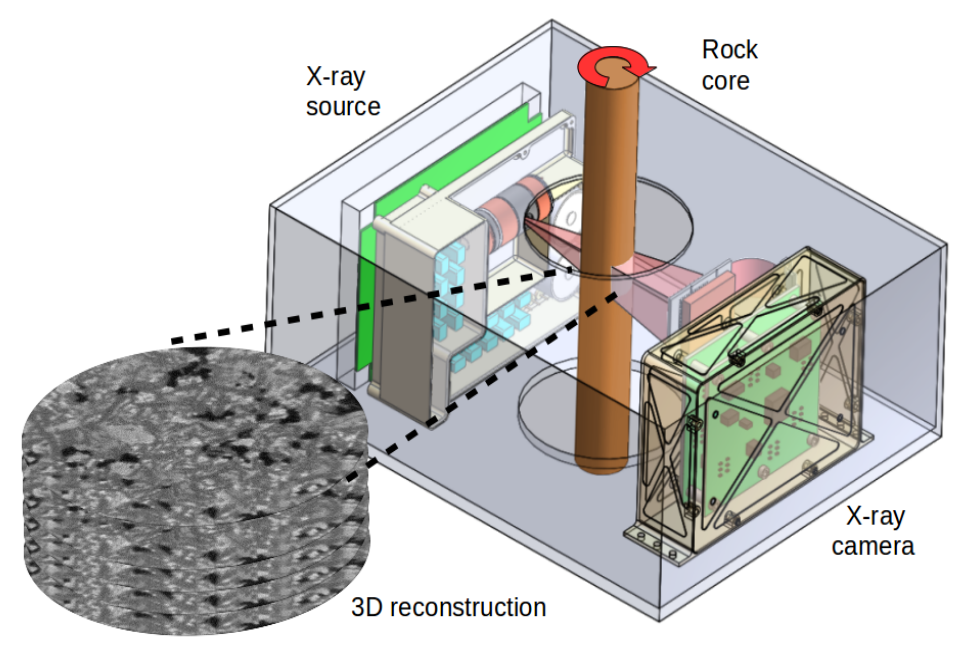

Summary: We propose the in situ deployment of high-resolution X-ray Computed Tomography instruments in the coming decade to non-destructively analyze the microstructure and composition of samples on rocky or icy bodies.

P. Sarrazin ${ }^{1}$, R. Obbard ${ }^{1}$, N. Vo ${ }^{2}$, K. Zacny ${ }^{3}$, N. Hinman ${ }^{4}$, B. Lafuente ${ }^{1}$, J. Bishop ${ }^{1}$, J. Chen ${ }^{5}$, D. Blake ${ }^{6}$, T. Bristow ${ }^{6}$, E. Rampe 7 , S. Byrne ${ }^{8}$, S. Eckley ${ }^{9}$

${ }^{1}$ SETI Institute, 189 Bernardo Ave, Mountain View CA 94043 psarrazin@seti.org,

${ }^{2}$ Diamond Light Source, Didcot, Oxfordshire OX11 0DE, UK,

${ }^{3}$ Honeybee Robotics, 398 W Washington Blvd., Pasadena, CA 91103,

${ }^{4}$ Department of Geosciences, University of Montana, Missoula MT 59812,

${ }^{5}$ Baja Technology, Tempe (AZ)

${ }^{6}$ NASA Ames Research Center, MS239-4, Moffett Field CA 94036.

${ }^{7}$ NASA Johnson Space Center, Houston, TX, USA

${ }^{8}$ Lunar \& Planetary Laboratory, University of Arizona, Tucson, AZ, USA

${ }^{9}$ Jacobs Technology, Johnson Space Center, Houston, TX 77058

Co-signers: D. Vaniman (PSI), A. Steele (Carnegie), A. Bramson (Purdue), I. Midtkandal (U. Oslo), A. Treiman (LPI), J. Blank (ARC), N. Cabrol (SETI), I. Smith (PSI), S. Morrison (Carnegie), B. Diamond (SETI), P. Becerra (U. Bern), D. Ming (JSC), R. Bonaccorsi (SETI), G. J. Taylor (U. HI), J. Farmer (ASU), R. Zeigler (JSC)

Co-author and Co-signer sign-up spreadsheet: https://docs.google.com/spreadsheets/d/1wwMmXaYrklZSdxNtRRRXolkKrwql1LYH4L 70qB QspM/edit?usp=sharing 


\section{THE POTENTIAL OF MICROCT FOR PLANETARY EXPLORATION}

Radiography is broadly used in the medical sector and in the industry for non-destructive analysis of multiphase materials. X-ray Computed Tomography (XCT) uses a series of 2-dimensional (2D) radiographic images collected from different orientations to compute a 3-dimensional (3D) model of a sample. Developed first for medical applications in the 70's, XCT soon found applications in geosciences for rapid and non-destructive analysis of the internal structure of rock samples [Ketcham 2001]. This technique has seen many applications in the analysis of planetary materials [Ebel 2007; Hanna 2017]. XCT resolution varies broadly depending on applications. MicroCT, a micron-scale XCT using a combination of focused X-ray sources (X-ray tube or synchrotron light source) and geometric magnification, has gained much interest in geology because of its ability to resolve individual grains or pores in rock samples. For instance, microCT has been used extensively in the study of depositional processes in sedimentary rocks [e.g. Falvard 2017] and more recently in ice [Iverson 2017].

The high-energy radiation used in radiography and XCT (20-400 keV depending on application) penetrates through the sample in its native state, with no need for preparation. This non-destructive analysis allows imaging internal features that could otherwise only be reached with highly-destructive serial sectioning of the sample. Furthermore, X-rays can penetrate through the walls of a sample container, such as the sealed break-off tube canisters that will host the Mars sample-return cores. MicroCT has been recommended as the $1^{\text {st }}$ technique to be applied to Mars samples received on Earth, while still in their sealed containers [Kminek 2014]. Risks of damage to Martian organics that might be present in the sample are being assessed as part of the preparation for Mars sample return [Welzenbach 2017, 2020].

Radiography and microCT are particularly pertinent techniques for in-situ non-destructive analysis of core samples in planetary exploration, but no miniature instrument has been developed for space flight. We are addressing this gap with the development of PIXI (Planetary In-situ X-ray Imager), an instrument that will offer on-board radiography, microCT, and optionally X-ray Fluorescence (XRF) to planetary exploration rovers or landers fitted with a sample coring drill, or a fetch rover for samplereturn missions. PIXI is currently developed to TRL 4 under SBIR funding [url1]. A flight PIXI will enable microCT on the inner planets, the Moon, asteroids or comets, and can be ready for deployment within the coming decade. In parallel, a variant of PIXI is being developed in a collaboration between the SETI Institute and Honeybee Robotics for in-situ analysis of Mars Polar Layer Deposit (PLD) under PICASSO 2019 funding ("Micro In Situ Tomography (MIST)”. PI: R. Obbard)[Obbard 2020].

\section{POSSIBLE DEPLOYMENTS.}

We envision four possible applications of in-situ microCT, summarized in Table 1, each leading to specific implementation, capabilities, and technical requirements.

Deployment scenario A: in situ microCT of ice cores. The water or $\mathrm{CO}_{2}$ matrix of ice cores has low linear X-ray absorption coefficient relative to rock-forming minerals, enabling radiography/microCT analysis at lower X-ray energies. This technique is used to analyze the location and geometry of liquid, gaseous, and mineral inclusions in terrestrial sea ice and ice sheets [Iverson 2017]. The feasibility of this application was successfully demonstrated based on available flight technologies: large CCD or CMOS cameras, scintillator, miniature microfocused X-ray tube and associated $25 \mathrm{kV} / 4 \mathrm{~W}$ power supply. While much remains to be done for the maturation of an ice microCT, there is no critical technical barrier to this development.

Deployment scenario B and C: in situ microCT/XRF of rock cores. Rock cores present a greater challenge than ice cores because of their much higher X-ray attenuation at low energies. Bare cores of 
small diameter (5-10 mm), or similar cores sealed in a low Z canister can be analyzed by microCT with higher energy sources. Figure 1 shows a notional design of a PIXI instrument. An X-ray tube with a sharp focus illuminates the sample in a cone beam geometry, and an X-ray camera collects attenuation images. The core is precisely rotated at small steps by a sample handling stage (not shown) to collect radiographic data from many different orientations. X-ray fluorescence (XRF) analysis can be obtained to measure the chemistry of the surface of the cores, with spatial resolution provided by means of a collimator or X-ray optic on a Silicon Drift Diode (SDD) detector to selectively measure the XRF emission of a small surface area. Rotation and translation of the core, necessary for the CT scan, enable building coarse elemental maps of the surface. These surface measurements can be correlated to the internal structure visible in the 3D reconstruction: veins across the core will be chemically analyzed at the surface, allowing extrapolation of the chemical composition of these internal features.

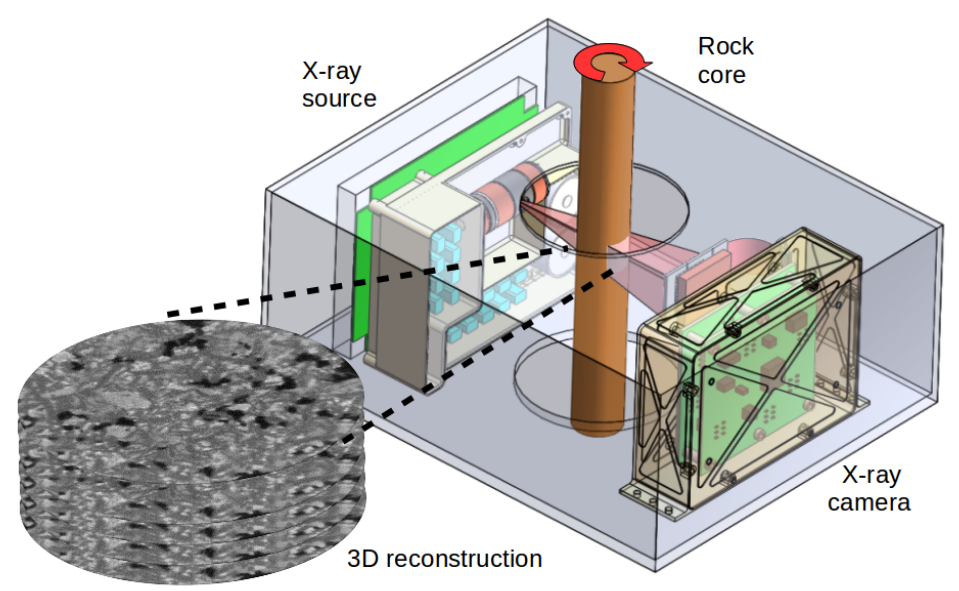

Deployment scenario D: in situ radiography of Mars 2020 return samples. In this scenario, the rock cores and canister specifications have already been set and are not optimized for in-situ microCT. For this implementation of PIXI, our main target is to collect high-resolution radiographic images of the cores inside the canister. We are investigating the potential of the instrument concept for XCT and 3D reconstruction of the sealed cores, but we don't regard this as a requirement; radiographic analyses of the cores through Figure 1: Notional design of a PIXI microCT instrument for rock their containers in one or a few orientations cores utilizing an X-ray camera with a scintillator coupled to a CCDwould already provide invaluable data or CMOS imager, or a hybrid-pixel detector for reduced noise and about the core integrity, its volume, additional energy discrimination, and an X-ray tube combined to a fragmentation state, etc., critically high-voltage power supply currently being developed to $50 \mathrm{kV}$.

Core handling and XRF module not represented. Anticipated dimensions $250 \times 200 \times 100 \mathrm{~mm}$. important for the core selection or documentation in the context of the fetchrover mission.

Table 1: Specifics of 4 possible scenarios of deployment of PIXI.

\begin{tabular}{|c|l|l|l|l|}
\hline \multicolumn{1}{|c|}{ Samples } & \multicolumn{1}{|c|}{ Applications } & \multicolumn{1}{|c|}{ Measurements } & \multicolumn{1}{|c|}{ Methods } \\
\hline A & $\begin{array}{l}\text { Ice core with mineral } \\
\text { and gas inclusions }\end{array}$ & $\begin{array}{l}\text { Robotic science on Mars } \\
\text { Polar Layered Deposits, } \\
\text { Europa, comets,... }\end{array}$ & $\begin{array}{l}\text { Dust inclusion, porosity, } \\
\text { layering, fracture. }\end{array}$ & Radiography, microCT. \\
\hline B & Bare rock core & $\begin{array}{l}\text { Robotic science missions } \\
\text { on Mars, the Moon, ... }\end{array}$ & $\begin{array}{l}\text { Core structure, layering, } \\
\text { veins, chemistry. }\end{array}$ & $\begin{array}{l}\text { Radiography, microCT, } \\
\text { XRF. }\end{array}$ \\
\hline C & $\begin{array}{l}\text { Rock core sealed in } \\
\text { Be or Al canister }\end{array}$ & $\begin{array}{l}\text { Future sample caching / } \\
\text { return missions }\end{array}$ & $\begin{array}{l}\text { Core structure, layering, } \\
\text { veins, fragmentation, } \\
\text { surface chemistry. }\end{array}$ & $\begin{array}{l}\text { Radiography, microCT, } \\
\text { possibly XRF. }\end{array}$ \\
\hline D & $\begin{array}{l}\text { Rock core sealed in Ti } \\
\text { canister }\end{array}$ & $\begin{array}{l}\text { Mars 2020 break-off-tubes } \\
\text { sorting and documenting } \\
\text { on fetch-rover }\end{array}$ & $\begin{array}{l}\text { Core volume \& } \\
\text { estimated mass, } \\
\text { fragmentation, } \\
\text { microstructure. }\end{array}$ & $\begin{array}{l}\text { Radiography (CT } \\
\text { probable, microCT } \\
\text { unlikely). }\end{array}$ \\
\hline
\end{tabular}




\subsection{In situ analysis of ice cores of Mars Polar Layered Deposits (PLD).}

Mars has a simplified terrestrial planet climate with no complications from vegetation, people, or oceans, thus understanding this climate and its changes over time is critical to understanding climate change on all terrestrial planets and assessing past Martian habitability. PLD are multi-kilometer thick sequences of dusty-ice layers thought to record previous climatic conditions much like Earth's ice sheets record terrestrial climate fluctuations in their stratigraphy. Deciphering this polar record is a major goal of Mars research [Byrne 2009]. A microCT combined with a coring drill would detail approximately 1000 martian years of climatic history for each $1 \mathrm{~m}$ core collected, measuring particulate inclusion size and organization, voids, overall stratigraphy, and fractures caused by the coring. With a static lander on the flat upper surface of the North PLD (NPLD), a $1 \mathrm{~m}$ core sampled by microCT would detail approximately the last 1000 martian years of climatic history. With a rover traversing a surface exposure of the NPLD interior, several $1 \mathrm{~m}$ cores would provide 1000-martian-year snapshots of the martian climate spread over 1.5 Myr. Extensive layered deposits also exist at Mars' South Pole. Similarly, buried ice has been observed at the mid-latitudes of Mars as seen by MRO shallow radar and ice-exposing impacts [Byrne 2009]. The ice-coring drill + microCT suite could directly serve landed missions to these Mars locations as well as landed missions to icy bodies of our solar system such as Europa or comets. This analytical capability is proposed in 2020 WP [Becerra 2020, Edwards 2020, Bramson 2020].

Ice core with inclusions were successfully analyzed with the PIXI breadboard prototype [Obbard 2020]. An advanced prototype coupled to an ice-coring drill will be demonstrated in FY2021.

\subsection{In situ analysis of bare rock cores}

Coring rocks gives access to geologic records unbiased by surface weathering and dust accumulation. MSL was originally planned with a coring drill, core observation deck and grinder, though all of these were descoped during the rover development, and replaced with a powdergenerating percussion drill. Mars 2020 will be the $1^{\text {st }}$ Mars rover capable of collecting cores at Mars, but unfortunately with no means to analyze the cores prior to their caching for eventual sample return.

In the context of future robotic science missions, an on-board PIXI would enable microCT and XRF characterization of a core to establish its stratigraphy, porosity, fracturing, alteration, grain size distribution, and phase distribution, prior to possible grinding for powder-based instruments (on MSL: CheMin XRD/XRF, SAM mass spectrometer). A combination of PIXI and existing microscopy, mineralogy and chemistry instruments would provide a Mars rover with true petrographic capabilities.

In addition to Mars, PIXI can be deployed on other terrestrial planets and rocky moons.

Figure 2 shows results of analyses performed with an early PIXI prototype compared to a laboratory microCT system. The additional surface XRF capability will be demonstrated in FY2021, as well as the benefits of added spectral discrimination capabilities offered by hybrid-pixel detectors.

\subsection{Astrobiology: reveal unique microstuctures of extinct life}

Potential martian hot spring deposits [Ruff 2020] are prime targets for astrobiological exploration because of the likelihood that life on Earth developed in hydrothermal environments, their ease of detection from orbit, and their high habitability and preservation potential [Cady 2018]. On Earth, hot spring microfacies display predictable changes in population along thermal and chemical gradients [Walter 1993]. Figure 3 shows an example of hot spring core analysis with PIXI using a sample collected at a subsurface depth of $0.25 \mathrm{~m}$ from the interior cliff face of Excelsior Geyser in Yellowstone National Park. The rock shows biofabrics from Calothrix-dominated microbial mats. This Cyanobacterium lives in alkaline-chloride hot springs $(\mathrm{pH} \sim 8)$ in temperatures $25-50^{\circ} \mathrm{C}$. These fossil 
microbial mats comprise dominantly opal-A, an amorphous type of silica that forms readily on microbial surfaces upon cooling of water in the hot spring runoff channels. Vertical fenestral fabric, also known as palisade fabric, is divided into sub-centimeter units that are separated by laminar sheets also comprising opal-A. Geochemical, physical, and hydrological processes control preservation of microfossils through time. If this fabric was found on Mars, it could be a microfossil and would strongly indicate the presence of extinct or extant life.

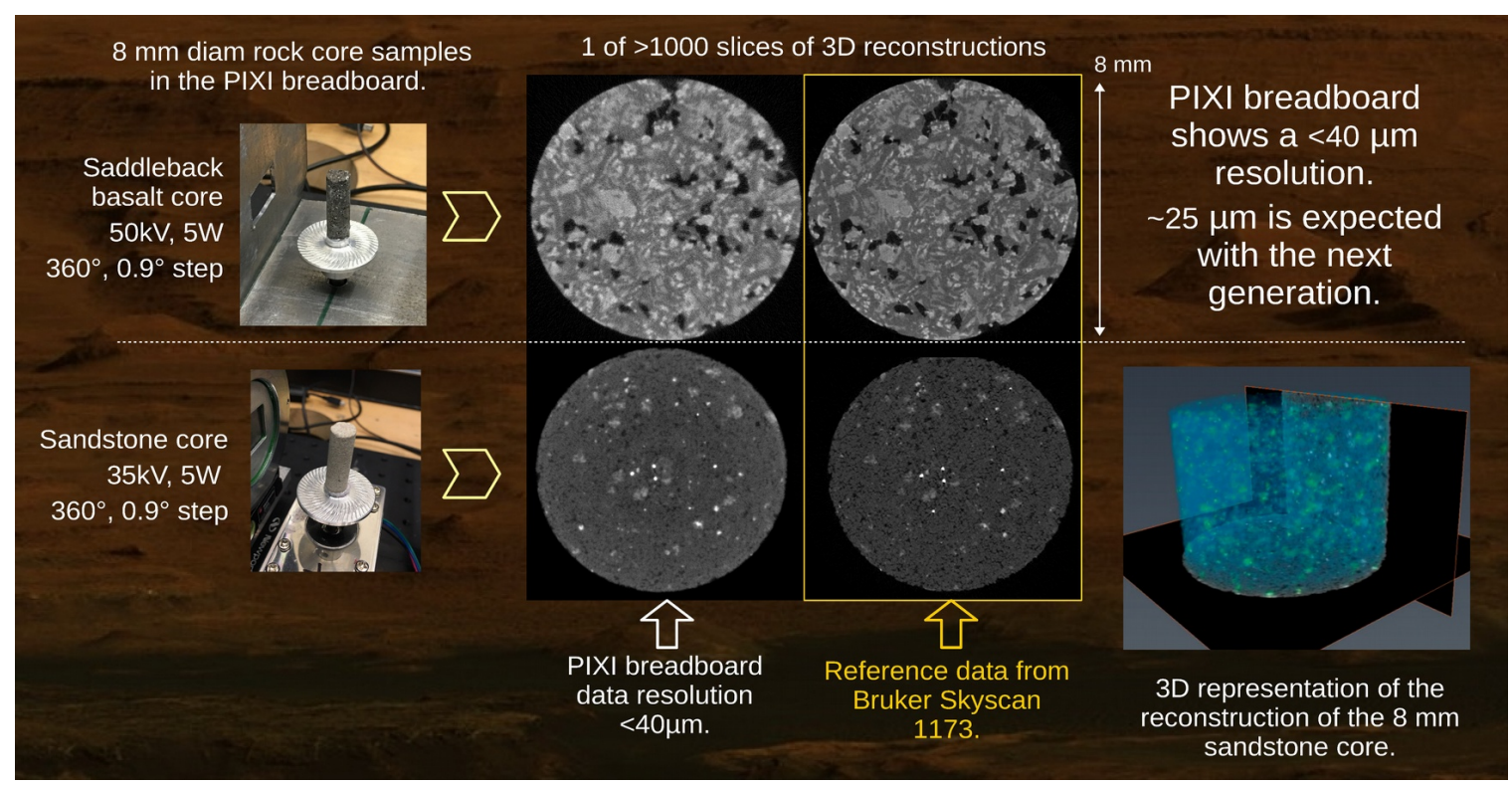

Figure 2: Examples of rock core data collected with the PIXI breadboard instrument with a Saddleback basalt core top, and sandstone core, bottom. Core diameter: $8 \mathrm{~mm}$. Central images show one slice of the 3D reconstructions (among 1000) ; PIXI slice (left) is compared to the equivalent slice of a laboratory microCT (right). Black areas: voids, lighter gray: higher absorption minerals.

Bottom right: 3D view of the PIXI reconstruction of the sandstone. The current $<40 \mu \mathrm{m}$ resolution in the $3 \mathrm{D}$ reconstruction is expected to be improved to about $25 \mu \mathrm{m}$.

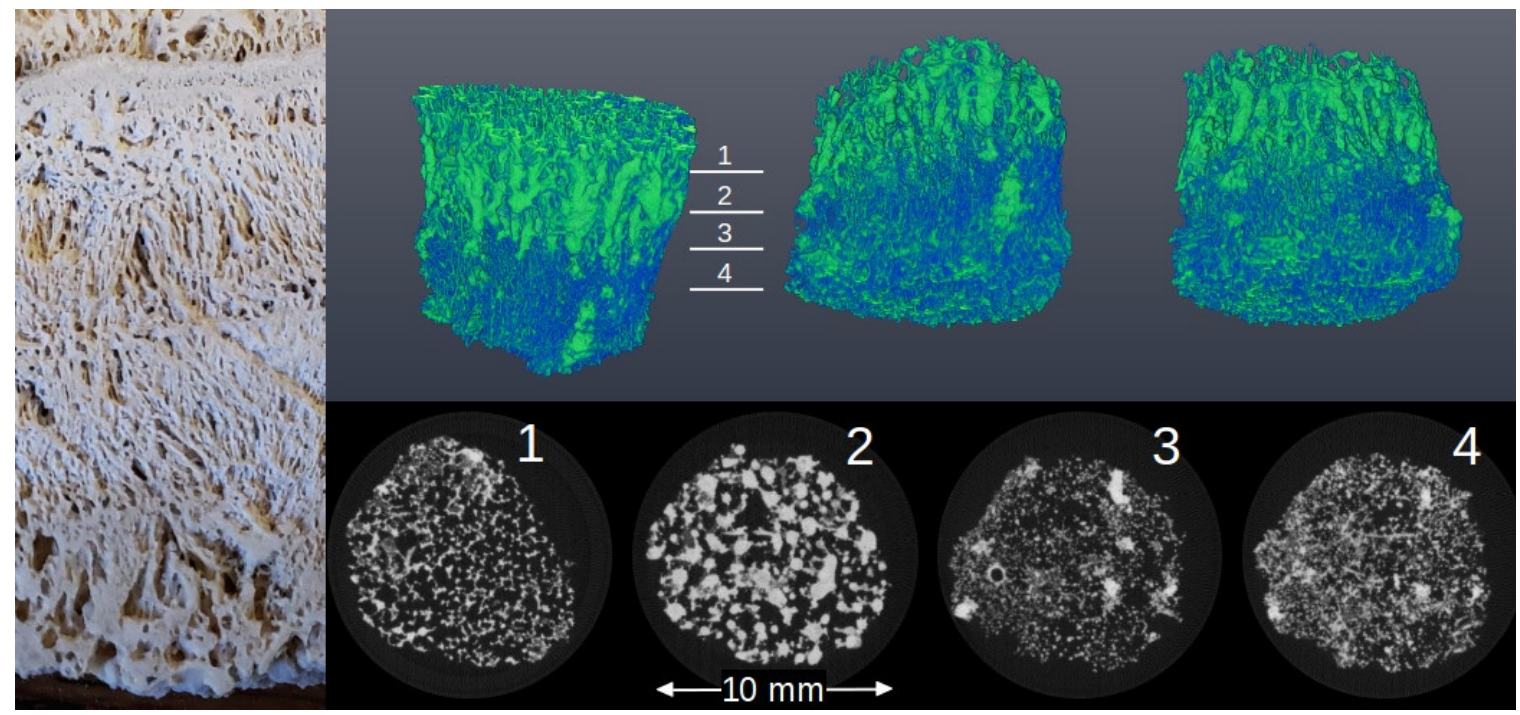

Figure 3: PIXI breadboard analysis of an Excelsior Geyser hot spring sample; left: photograph of a vertical section; top: rendering of the $3 \mathrm{D}$ reconstruction; bottom: horizontal slices of the same data show the structures of the opal-A coatings. 


\subsection{In situ analysis of rock cores sealed in canisters}

Planetary science strategy described in the last Decadal Survey has put a strong emphasis on Mars sample return over robotic science. In continuation of this strategy, a PIXI combining microCT and XRF will be particularly pertinent in the context of core sample caching or sample return missions beyond Mars 2020. Using sealed canisters specifically designed to enable a complete X-ray analysis, PIXI could analyze the core immediately after its collection to assess its integrity, volume, estimated mass, chemistry, stratigraphy, etc. This unprecedented capability would provide more effective sampling and critical documentation of the core state at the time of caching. The PIXI data would complement the terrestrial analysis of as-received samples. Indeed, microCT is proposed as the $1^{\text {st }}$ technique to be applied to Mars samples received on Earth, because of its ability to non-destructively analyze the core through the canister [Kminek 2014].

\subsection{Documenting and sorting of Mars 2020 break-off tubes.}

Mars 2020 will be able to collect up to 37 cores, but very limited data will be available about the cores, the rover instrument suite focusing its analyses on the rock from which the cores are collected, rather than the cores themselves. A PIXI-instrumented fetch rover would be capable of probing the cores through their canisters. Figure 4 shows radiographic images collected with PIXI using a Mars 2020 break-off tube reproduction and a range of $13 \mathrm{~mm}$ diameter rock cores. These images clearly show the cores through the Ti tube walls. No XRF capability is possible through the Ti wall due to the absorption of all useful fluorescence signal. PIXI would provide radiographic data from different directions to assess the core volume, fragmentation state, layering, grain-size distribution and multiphase structure. XCT has not been demonstrated yet in these conditions, but might be possible with limited performance relative to the applications previously described. Whether simple radiographic images or a full set of XCT data with 3D reconstruction, the data would be critical in characterizing the samples in detail from the rover. The handling of the Mars 2020 cores by the fetch rover and the launch from Mars and return trip to Earth are likely to agitate sediments in the tubes such that the original sedimentary structures are lost by the time they are received on Earth. No other technique can better guide a core selection and document the state of the cores prior to their launch from Mars. The PIXI data would be directly compared to the state of the core as received on Earth, analyzed by laboratory or synchrotron microCT instruments.
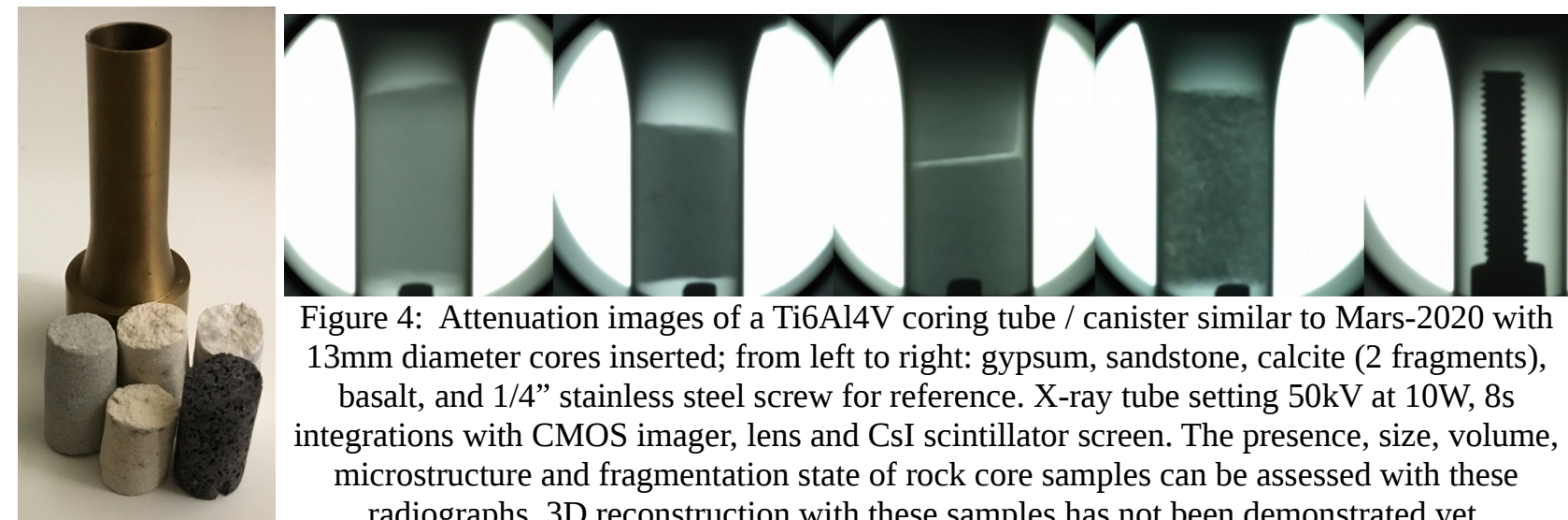

Figure 4: Attenuation images of a Ti6Al4V coring tube / canister similar to Mars-2020 with 13mm diameter cores inserted; from left to right: gypsum, sandstone, calcite (2 fragments), basalt, and 1/4” stainless steel screw for reference. X-ray tube setting $50 \mathrm{kV}$ at $10 \mathrm{~W}, 8 \mathrm{~s}$ integrations with CMOS imager, lens and CsI scintillator screen. The presence, size, volume, microstructure and fragmentation state of rock core samples can be assessed with these radiographs. 3D reconstruction with these samples has not been demonstrated yet.

\subsection{Analysis of rock fragments and regolith.}

All planetary applications described above assume the rover or lander is capable of collecting a core (or using a core already prepared). The PIXI instrument is, however, not limited to core samples. A rock fragment commensurate with the core diameters envisioned $(5-15 \mathrm{~mm})$ could also be analyzed to 
provide a 3D model of its outer shape, its internal structure and its surface composition. Likewise, regolith samples in tubular containers could be analyzed for ISRU exploration. The instrument would fit one of the upcoming Commercial Lunar Payload Services (CLPS) missions.

\section{TECHNICAL CHALLENGES}

PIXI is based on a simple design that doesn't require new technology to be developed. The barrier to deployment of PIXI in space is well understood and addressed with its development program.

\subsection{Higher energy X-ray illumination vs high voltage.}

PIXI relies on a microfocused X-ray tube for the sample illumination. Resolution $<40 \mu \mathrm{m}$ has been demonstrated using $60 \mu \mathrm{m}$ X-ray spots, and $<25 \mu \mathrm{m}$ is expected with new X-ray tubes with a $30 \mu \mathrm{m}$ focus. This X-ray focal spot size is routinely achieved with simple X-ray tube architectures.

The energy of illumination must be sufficient to penetrate through the sample. We demonstrated that $25 \mathrm{kV}$ is sufficient for application to $25 \mathrm{~mm}$ diameter ice cores. X-ray sources such as in planetary XRD instruments ( $30 \mu \mathrm{m}$ focus, $25 \mathrm{kV}, 5 \mathrm{~W}$ ) satisfy the requirements for ice core analysis with a simple change to a tungsten anode. PIXI analysis of rock cores has been demonstrated with the tube set at up to $50 \mathrm{kV}$, a leap from the $25 \mathrm{kV}$ demonstrated on Mars. A key element of the PIXI development is the design of an X-ray source capable of $50 \mathrm{kV}$ X-ray generation, but based on a bipolar X-ray tube to limit the HVPS requirement: the filament-based cathode is polarized at $-15 \mathrm{kV}$ and the anode (target) polarized at up to $+35 \mathrm{kV}$. This development is done in partnership with RTW (Germany) for the miniature bipolar X-ray tube, and Battel Engineering (AZ) for the flight HVPS.

\subsection{X-ray camera.}

PIXI requires a high-resolution imager with good quantum efficiency (QE) up to $50 \mathrm{keV}$ for the collection of absorption images. The most conventional method uses a scintillator film coupled to a visible light camera (CCD or CMOS) collecting the fluorescence emission of the scintillator under Xray excitation. The PIXI breadboard data shown here were collected with columnar CsI film and CMOS sensors, coupled via a 1:1 fiber optic plate or a lens. We are also evaluating hybrid-pixel detectors, coupling a GaAs or CdTe sensor chip with a CMOS readout chip, that provide very high QE, very low noise, single photon sensitivity, high frame rate, no cooling requirement, and energy discrimination that could broaden the analytical capabilities of PIXI. Current hybrid detectors offer slightly lower spatial resolution than scintillator-based cameras. Neither technology presents a technological barrier to planetary deployment, but will require maturation efforts.

\subsection{Scanning.}

XCT requires a precisely controlled rotation of the sample relative to the X-ray source and camera. This can be achieved by rotating the instrument around a fixed sample or maintaining the instrument fixed in position and rotating the sample. The former approach is implemented in the MIST prototype that combines ice coring and microCT scanning, while the latter is the base of the rock core PIXI concept, using a core handling stage under development at Honeybee Robotics. These two configurations require substantial mechanical design efforts, but neither oppose a technology gap.

\subsection{Volume reconstruction}

Data processing pipeline for PIXI combines preprocessing and reconstruction methods with a dedicated Python package [Vo 2019]. Preprocessing steps include flat-field correction, beam hardening correction, zinger removal, and ring artifact removal. The cone-beam reconstruction methods for GPU are optimized for complex misaligned systems. They are based on the filtered back-projection (FBP) method and back-projection filtering (BPF) method incorporating all geometric parameters of a conebeam system. These parameters are: the pitch, roll, yaw, and positions of a rotation axis; the X-ray 
source positions; and the source-detector distance. The geometric parameters are calibrated by analyzing the trajectory of a point-like sample (e.g. a sphere) at two heights. In a planetary deployment context, the complex processes are currently assumed to involve ground based computing, but we are evaluating options for partial or complete on-board processing to limit downlink requirements.

\section{CONCLUSION}

Technology is being developed to enable high-resolution X-ray computed tomography in landed missions on rocky or icy bodies in the coming decade. The Planetary In-situ X-ray Imager PIXI will be sufficiently miniaturized to be hosted in a mid-sized rover fitted with a robotic arm for sample collection. PIXI has been demonstrated at TRL-4 and could be rapidly matured for flight as it inherits most components from planetary instruments already flown or under mid-TRL maturation program.

\section{REFERENCES}

- Becerra P. et al., WP 2020, The Importance of the Climate Record in the Martian Polar Caps.

- Bramson A. et al. WP 2020, Mid-Latitude Ice on Mars: A Science Target for Planetary Climate Histories and an Exploration Target for In Situ Resources.

- Byrne, S. (2009) The polar deposits of Mars. Annual Review of Earth and Planetary Sciences, 37, 535560, doi:10.1146/annurev.earth.031208.100101.

- Cady, S.L. et al. (2018) From Habitability to Life on Mars (pp. 179-210), Elsevier,

- Ebel, Denton S., and Mark L. Rivers. "Meteorite 3-D synchrotron microtomography: Methods and applications." Meteoritics \& Planetary Science 42, no. 9 (2007): 1627-1646.

- Edwards C. et al., WP 2020, Emerging Capabilities for Mars Exploration.

- Falvard, S. and Paris, R. (2017) X-ray tomography of tsunami deposits: Towards a new depositional model of tsunami deposits. Sedimentology, 64(2), 453-477.

- Hanna, Romy D., and Richard A. Ketcham. "X-ray computed tomography of planetary materials: A primer and review of recent studies." Geochemistry 77, no. 4 (2017): 547-572.

- Iverson N. et al. The first physical evidence of subglacial volcanism under the West Antarctic Ice Sheet. Scientific Reports 7: 11457 (2017) DOI: 10.1038/s41598-017-11515-3

- Ketcham R.A and Carlson, W.D. (2001) Acquisition, optimization and interpretation of X-ray computed tomographic imagery: applications to the geosciences. Comp. \& Geosc., 27, 381-400.

- Kminek, G., et al. (2014) Report of the workshop for life detection in samples from Mars. Life Sciences in Space Research, 2, 1-5.

- Obbard, R.W. et al., (2020) In-situ microCT instrument for the North Polar Layered deposits of Mars, 2020 ICMPSE https://www.hou.usra.edu/meetings/marspolar2020/pdf/6078.pdf

- Ruff, S. W. , K. A. Campbell, M. J. Van Kranendonk, M. S. Rice, J. D. Farmer (2020), The Case for Ancient Hot Springs in Gusev Crater, Mars, https://doi.org/10.1089/ast.2019.2044

- url\#1: http://examinart.com/pixi/

- Vo N. T., R. Atwood, M. Drakopoulos, (2019) Preprocessing techniques for removing artifacts in synchrotron-based tomographic images, https://doi.org/10.1117/12.2530324

- Walter, M.R. and Des Marais, D.J., (1993) Preservation of Biological Information in Thermal Spring Deposits: Developing a Strategy for the Search for Fossil Life on Mars. Icarus, 101(1), pp.129-143.

- Welzenbach, L.C. et al. (2017) X-Ray Computed Tomography: The First Step in Mars Sample Return Processing. 48th Lunar and Planetary Science conference, 20-24 March, Houston, TX.

- Welzenbach, L.C. et al. (2020) "Potential Alteration of Analogue Regolith by X-Ray Computed Tomography." LPSC 2326 (2020): 2500. 